Article

\title{
Design of Dual-Band Terahertz Perfect Metamaterial Absorber Based on Circuit Theory
}

\author{
Zhongmin Liu ${ }^{1,2}$, Liang Guo ${ }^{1,2}$ and Qingmao Zhang ${ }^{1,2, *}$ \\ 1 Guangzhou Key Laboratory for Special Fiber Photonic Devices and Applications, \\ South China Normal University, Guangzhou 510006, China; Liuzm@m.scnu.edu.cn (Z.L.); \\ guoliangchn@163.com (L.G.) \\ 2 Guangdong Provincial Key Laboratory of Nanophotonic Functional Materials and Devices, \\ South China Normal University, Guangzhou 510006, China \\ * Correspondence: zhangqm@scnu.edu.cn; Tel.: +86-1366-031-7109
}

Received: 30 July 2020; Accepted: 5 September 2020; Published: 8 September 2020

check for updates

\begin{abstract}
We present a novel strategy for designing a dual-band absorber based on graphene metasurface for terahertz frequencies. The absorber consists of a two-dimensional array of patches deposited on a metal-backed dielectric layer. Using an analytical circuit model, we obtain closed-form relatinos for the geometrical parameters of the absorber and the properties of the applied materials to achieve the dual-band absorber. Two absorption bands with perfect absorption at the preset frequencies of 0.5 and $1.5 \mathrm{THz}$ are achieved. The results obtained by the analytical circuit model are compared to the simulations carried out by full-wave electromagnetic field analysis. The agreement between results is very good. We demonstrate that the graphene absorber remains as the dual band for a wide range of the chemical potential. Furthermore, the recommended dual band absorber is insensitive in terms of polarization and remain within various incident angles.
\end{abstract}

Keywords: dual-band absorber; graphene metasurface; circuit model

\section{Introduction}

Metamaterials are artificially-made media with uniquely engineered electric permittivities and magnetic permeabilities [1,2] and enabled design of thin electromagnetic energy absorbers [3]. Metamaterial-based absorbers have attracted considerable attention in the terahertz $(\mathrm{THz})$ region due to their promising applications in imaging [4], biosensing [5,6], optical control [7], etc. Recently, the realization of dual-band terahertz absorbers based on metamaterials has been investigated [8-14]. Such dual-band absorbers can be utilized in dual-band transceiver systems and enhance energy absorption for imaging and biochemical sensing. In these structure, various mechanisms of dual-band absorption have been recommended by a combination of multiple resonators with a hybrid structure metamaterial layer or stacked structure metamaterial layers. However, these works require not only the structure of the complex, but also the corresponding tedious design and fabrication process. Therefore, designing simple structures with an efficient method is of great importance [15-19].

To realize metamaterial-based absorbers in the low $\mathrm{THz}$ region, one can use the graphene patterned arrays. Graphene, consisting of a two-dimensional layer of carbon atoms arranged in a honeycomb lattice, has recently been demonstrated as one of the best materials for many applications in electrical and photonic devices due to its exotic properties [20-23], such as high optical transparency [24], ultrafast electronic transport [25], and controllable electric conductivity, which can be tuned by changing the chemical potential [26]. Furthermore, graphene has the ability to supporting surface plasmon polaritons in the terahertz and infrared ranges [27]. In addition, in comparison to surface plasmon 
polaritons in noble metals, graphene plasmon polaritons display greater ability to achieve high local field, subdiffraction confinement, and low optical losses [28,29].

In this paper, a simple structure, composed of a period array of graphene patches deposited on a dielectric spacer terminated by a metallic back reflector, is used to design a dual-band $\mathrm{THz}$ absorber. We propose a circuit model to extract the geometrical parameters of the absorber and the properties of the material in the closed-form. Furthermore, we present a simple and effective approach for achieving perfect absorption at two preset frequencies. The input admittance of the absorber at the two frequencies is designed to match the admittance of free-space. Moreover, the imaginary part of the input admittance at the central frequency is set by applying an additional condition to be zero. To validate the approach, dual-band perfect $\mathrm{THz}$ absorbers have been investigated and designed. Then, full-wave simulations are used to confirm the design method. Excellent agreements have been achieved. It should be noted that the recommended absorber is polarization insensitive for normal incident electromagnetic waves owing to its symmetric structure. Besides, both dual-band and the high absorption effectiveness are affected slightly even at high angles of incidence for both TE and TMpolarizations.

The paper is organized as follows. The equivalent circuit model of the proposed device is introduced, and then a design scheme leading to an dual-band absorber is proposed in Section 2. Numerical validation of the accuracy and effectiveness of the model as well as discussions are presented in Section 3. Finally, conclusions are drawn in Section 4.

\section{Design Method}

The geometry of the proposed absorber is schematically shown in Figure 1, in which the top layer of periodic array of graphene patches with period $D$ and gap $g$ between the patches and the bottom layer of a reflective golden plate with finite conductivity of $4 \times 10^{7} \mathrm{~S} / \mathrm{m}$ are separated by a dielectric spacer with relative permittivity $\varepsilon_{d}$ and thickness $d$. It is worth mentioning that in the fabrication process, the $\mathrm{Si}$ is used to support the graphene patches. The Au film is deposited on the $\mathrm{Si}$ bottom by gilding. Next, a continuous and uniform atomic monolayer of graphene can be grown by the chemical vapor deposition method, then patterned into closely packed square patch arrays using e-beam lithography.

(a)

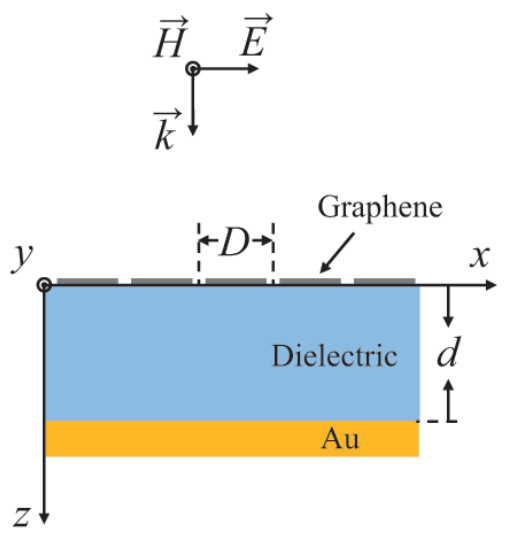

(b)

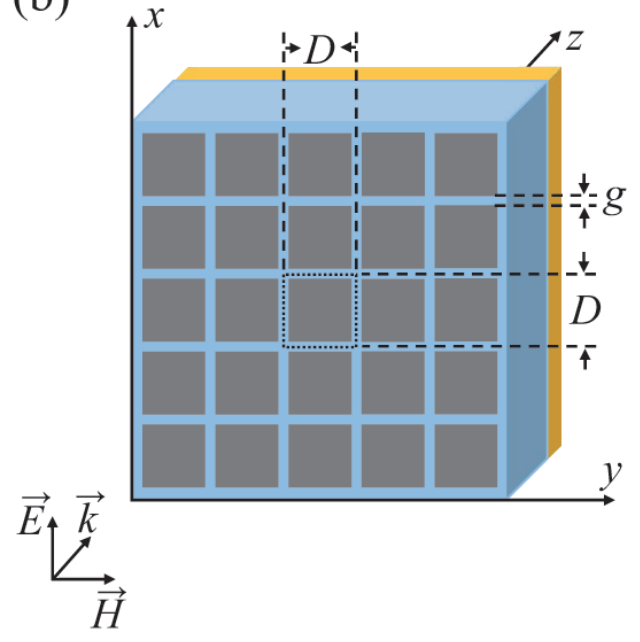

Figure 1. Schematic diagram of the proposed absorber: (a) cross section view and (b) 3D view.

Based on transmission line theory, the whole structure can be modeled as circuit elements [30]. The equivalent circuit of the proposed structure at normal incidence for TM polarization (electric field along the $x$ direction) is demonstrated in Figure 2. The graphene patch array can be modeled by a shunt admittance $Y_{g}$. The homogeneous regions namely the free space, the dielectric spacer and the 
Au backplate are modeled as transmission lines with the admittances $Y_{0}, Y_{d}$ and $Y_{A u}$, respectively. It should be pointed out that the metallic back reflector which is suppressing the transmittance can be approximately considered as a short circuit.

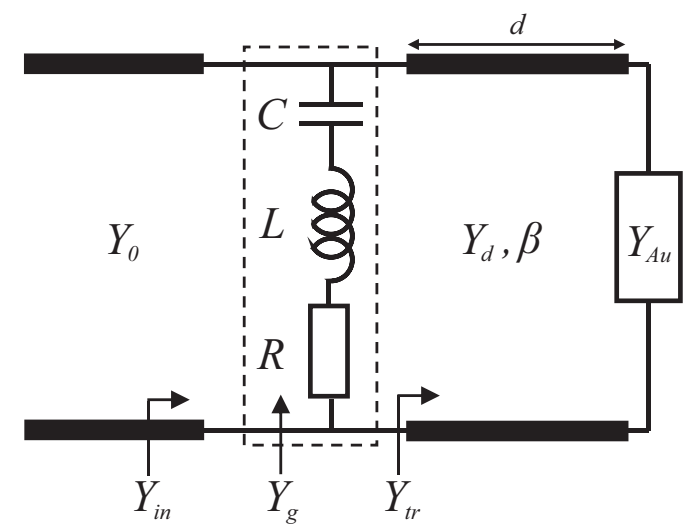

Figure 2. Equivalent circuit model for the graphene metasurface-based absorber.

An accurate circuit model has been proposed by Barzegar-Parizi et al. for a graphene patch array illuminated by a normally incident plane wave [31]. The admittance $Y_{g}$ in the model represents an infinite number of parallel R-L-C circuits, each corresponding to a mode of the structure.

$$
Y_{g}=\sum_{n=1(o d d)}^{\infty}\left(R_{n}+j \omega L_{n}+\frac{1}{j \omega C_{n}}\right)^{-1}
$$

in which $\omega=2 \pi f$ shows the angular frequency and the values of $R_{n}, L_{n}$, and $C_{n}$ are calculated as

$$
R_{n}=\frac{D^{2} K_{n}}{S_{n}^{2}} \operatorname{Re}\left\{\sigma_{g}^{-1}\right\}, L_{n}=\frac{D^{2} K_{n}}{S_{n}^{2}} \frac{\operatorname{Im}\left\{\sigma_{g}^{-1}\right\}}{\omega}, C_{n}=\frac{S_{n}^{2}}{D^{2} K_{n}} \frac{2 \varepsilon_{e f f}}{q_{n}}
$$

where $S_{n}=\int_{S} \xi_{n}(x, y) \mathrm{d} S, K_{n}=\int_{S}\left|\xi_{n}(x, y)\right|^{2} \mathrm{~d} S$, in which $S$ is the surface of a patch, $\xi_{n}(x, y)$, and $q_{n}$ are the $\mathrm{n}$ 'th normalized eigenfunction and eigenvalue corresponding to $\mathrm{n}$ 'th the resonant modes excited by the array of the patches. $\varepsilon_{e f f}=\varepsilon_{0}\left(1+\varepsilon_{d}\right) / 2$ is the average permittivity of the mediums surrounding the graphene patches, $\sigma_{g}$ is the surface conductivity of the graphene.

It is known that graphene's conductivity is derived using the Kubo formula, which is described with interband and intraband contributions [32,33]. For the $\mathrm{THz}$ frequencies, the photon energy $\hbar \omega<<2 \mu_{c}\left(\mu_{c}\right.$ is the chemical potential), the interband part is negligible comparing to the intraband [33, 34]. Therefore, graphene can be described by the Drude-like surface conductivity in the THz range:

$$
\sigma_{g}=\frac{\sigma_{0}}{1+j \omega \tau}
$$

where

$$
\sigma_{0}=\frac{e^{2} k_{B} T \tau}{\pi \hbar^{2}}\left\{\frac{\mu_{c}}{k_{B} T}+2 \ln \left(e^{-\mu_{c} / k_{B} T}+1\right)\right\}
$$

where $e$ is the electron charge, $k_{B}$ is Boltzmann's constant, and $\hbar$ is the reduced Planck's constant. $T$ is the temperature (fixed to $300 \mathrm{~K}$ ) and $\tau$ is the relaxation time of graphene.

Designing the proposed absorber near the first resonance frequency of graphene patches, we can only consider the first mode and neglect the effect of higher-order modes. To avoid numerical calculation of the eigenvalues in the previous capacitance expression, we propose to replace the expression with the analytical capacitance admittance of metal patches proposed in [35]. Therefore, the surface admittance $Y_{g}$ of the graphene patches can be rewritten as follows, 


$$
Y_{g}=\left(R+j \omega L+\frac{1}{j \omega C}\right)^{-1}
$$

where

$$
R=\frac{1.13 D^{2}}{(D-g)^{2}} \operatorname{Re}\left\{\sigma_{g}^{-1}\right\}, L=\frac{1.13 D^{2}}{(D-g)^{2}} \frac{\operatorname{Im}\left\{\sigma_{g}^{-1}\right\}}{\omega}, C=\frac{2 \varepsilon_{e f f}}{\pi} \operatorname{Dln}\left\{\csc \left(\frac{\pi g}{2 D}\right)\right\}
$$

In the equivalent circuit model of Figure 2. $\beta=n k_{0}$ and $Y_{d}=n Y_{0}$ are the propagation constant and the admittance of transmission line matching the dielectric spacer, respectively, where $k_{0}=\omega / c$ (c is the speed of light) is the wavenumber of free space and the free-space admittance $Y_{0}=1 /(120 \pi)$. Therefore, the total input admittance of the device is obtained as

$$
Y_{i n}=Y_{g}-j Y_{d} \cot (\beta d)
$$

Suppose perfect absorption can be achieved at two frequencies $\omega_{1}$ and $\omega_{2}\left(\omega_{1}<\omega_{2}\right)$. Accordingly, the conditions for this to happen can be summarized as

$$
\begin{aligned}
& \left.\operatorname{Im}\left(Y_{i n}\right)\right|_{\omega=\omega_{1}}=0 \\
& \left.\operatorname{Re}\left(Y_{i n}\right)\right|_{\omega=\omega_{1}}=Y_{0}
\end{aligned}
$$

and

$$
\begin{aligned}
& \left.\operatorname{Im}\left(Y_{i n}\right)\right|_{\omega=\omega_{2}}=0 \\
& \left.\operatorname{Re}\left(Y_{i n}\right)\right|_{\omega=\omega_{2}}=Y_{0}
\end{aligned}
$$

We consider a central frequency defined as $\omega_{0}=\left(\omega_{1}+\omega_{2}\right) / 2$ and apply an additional condition on the structure by setting $\left.\beta\right|_{\omega=\omega_{0}} d=\pi / 2$. The condition leads to $L C=\omega_{1} \omega_{2}$. Thus, the resonance frequency of the graphene array can be realized between dual band. Then, the thickness of the dielectric slab is obtained as

$$
d=\frac{\pi c}{2 \sqrt{\varepsilon_{d}} \omega_{0}}
$$

Then, the admittance of the graphene array is seen as the input admittance of the structure, and it can be tuned to be matched to the free-space admittance near its resonant frequency [36]. After straightforward mathematical manipulations, from Equations (8) and (9) we obtain

$$
\tau=\frac{\sqrt{\varepsilon_{d}} \cot \left(\frac{\pi \omega_{1}}{\omega_{2}+\omega_{1}}\right)}{\omega_{2}-\omega_{1}}
$$

Choosing the two frequencies $\omega_{1}$ and $\omega_{2}$, one can obtain the relaxation time of graphene by Equation (11) for given values of $n$. The relaxation time can be tuned by the chemical potential through the relation $\tau=\left(\mu \mu_{c}\right) /\left(e v_{f}^{2}\right)$, in which $v_{f}=10^{6} \mathrm{~m} / \mathrm{s}$ is the Fermi velocity and $\mu$ is the electron mobility ranging from approximately $0.03 \mathrm{~m}^{2} / \mathrm{Vs}$ to $6 \mathrm{~m}^{2} / \mathrm{Vs}$, depending on the fabrication process [37-41]. Therefore, by computing the relaxation time, the chemical potential of graphene is computed by

$$
\mu_{c}=\frac{\tau e v_{f}^{2}}{\mu}
$$

At the end, the geometrical parameters of the structure are computed by Equations (6)-(10) as

$$
R=\frac{1}{Y_{0}\left(1+\tau^{2}\left(\omega_{2}-\omega_{1}\right)^{2}\right)} \Rightarrow \frac{g}{D}=1-\sqrt{\frac{1.13 Y_{0}\left(1+\tau^{2}\left(\omega_{2}-\omega_{1}\right)^{2}\right)}{\sigma_{0}}}
$$




$$
L C=\frac{1}{\omega_{1} \omega_{2}} \Rightarrow D=\frac{\pi \sigma_{0}}{2.26 \tau \varepsilon_{e f f} \omega_{1} \omega_{2}}\left(1-\frac{g}{D}\right)^{2} \ln ^{-1}\left\{\csc \left(\frac{\pi g}{2 D}\right)\right\}
$$

\section{Numerical Results and Discussion}

With the aid of the analytical equations presented in Section 2, dual-band perfect graphene absorbers are designed in this part. The performances of the designed absorbers are verified numerically using full-wave simulations (Lumerical Nanophotonic FDTD simulation software). Suppose the relative permittivity of the dielectric spacer is $\varepsilon_{d}=11.9$ corresponding to $\mathrm{Si}$ and the frequencies of the first band and second band are $f_{1}=0.5 \mathrm{THz}$ and $f_{2}=1.5 \mathrm{THz}$, respectively. Therefore, the central frequency is calculated as $f_{0}=1 \mathrm{THz}$. The value of the relaxation time is given as $\tau=5.49 \times 10^{-13} \mathrm{~s}$ from Equation (11). The electron mobility is assumed to be $\mu=0.75 \mathrm{~m}^{2} /$ Vs. Thus, using Equation (12), we have $\mu_{c}=0.732 \mathrm{eV}$. The thickness of the dielectric spacer is calculated by Equation (10) as $d=21.73$ $\mu \mathrm{m}$. Finally, from Equations (13) and (14), the patch width and the period are extracted as $g=2.93 \mu \mathrm{m}$ and $D=30.54 \mu \mathrm{m}$, respectively. With these procedures, a dual-band perfect absorber is designed.

The absorption spectra of the structure are plotted in Figure 3a, calculated from the equivalent circuit model approach and compared with that obtained from FDTD simulation. An excellent agreement is observed between the two results, demonstrating the equivalent circuit model is accurate and effective. Figure $3 \mathrm{~b}$ reveals the real and imaginary parts of the normalized input admittance as a function of frequency for the absorber. As it can be observed, the imaginary part of the normalized input admittance is zero at the two frequencies of $f_{1}$ and $f_{2}$, whereas the real part matches free space admittance perfectly. Therefore, the prepared conditions is fulfilled for impedance matching at two bands and a perfect dual-band absorption is achieved. All key material and geometrical parameters of the absorber can be calculated directly from the formulas proposed according to design requirements. The equivalent circuit model approach speeds up the absorber design and analysis.

(a)

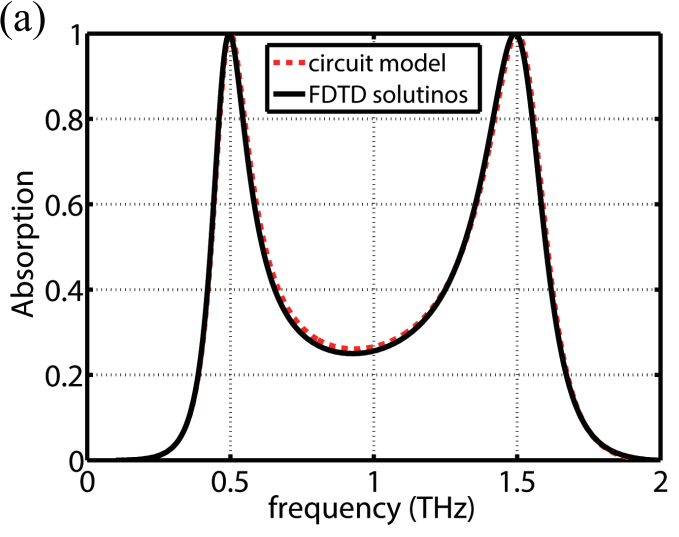

(b)

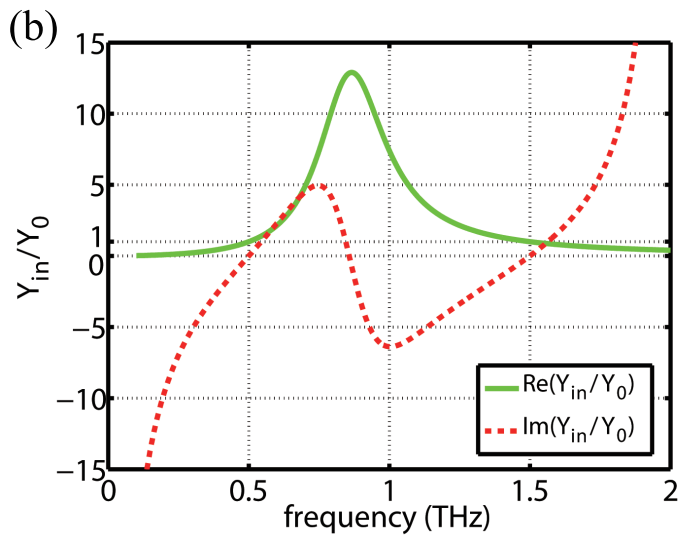

Figure 3. (a) The absorption spectrum of the designed dual-band absorber with the two frequencies at $0.5 \mathrm{THz}$ and $1.5 \mathrm{THz}$ calculated by the circuit model and FDTD simulation. (b) The real and imaginary parts of the normalized input admittance of the designed absorber.

It should be pointed out that there are slight disagreements between the circuit model and FDTD simulation. These slight disagreements result from neglecting the effect of higher-order modes of graphene patches in the circuit model shown in Figure 2, which was used in the design process. However, the effect of the higher-order modes has been included in the simulations.

Now, the tunability of the proposed device is investigated by changing the chemical potential of graphene. Figure 4 shows the absorption spectra for the chemical potential ranging between $0.4 \mathrm{eV}$ and $0.6 \mathrm{eV}$. It can be observed that the first resonant frequency is slightly shifted to a higher frequency region with increasing the chemical potential, whereas the absorption changes considerably. 
However, the location of the second resonant band moves to higher frequencies significantly with increasing the chemical potential while its absorption peak changes slightly.

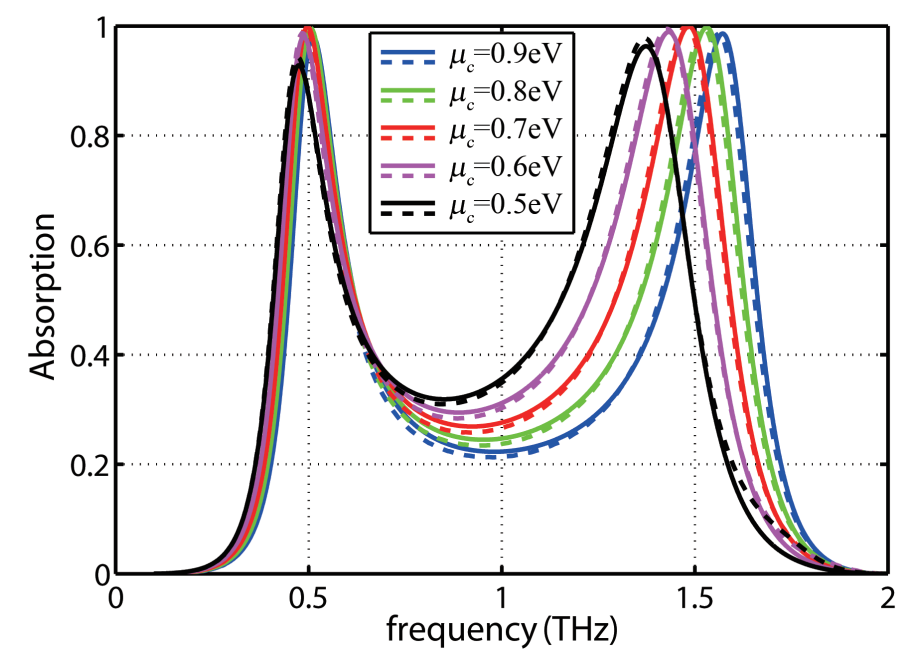

Figure 4. The absorption spectrum of the graphene-based proposed absorber for different chemical potential of graphene.

The polarization insensitive performance for the proposed absorber is important in practical applications. We plot the absorption spectra as a function of polarization angle and frequency in Figure 5. Due to the symmetrical design of the structure, it is clearly seen that the absorption spectra of the proposed dual-band absorber is polarization insensitive.

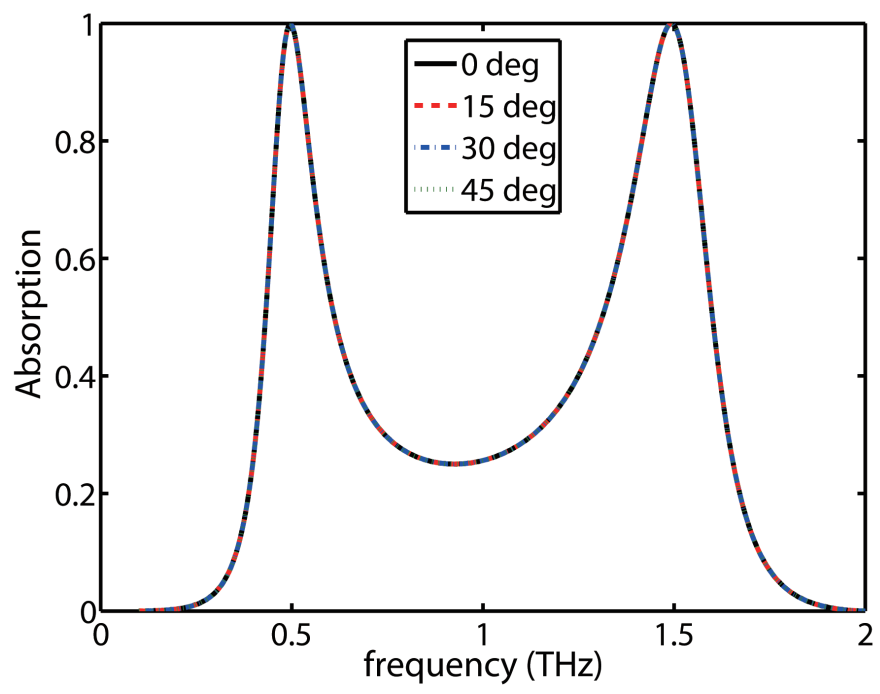

Figure 5. The absorption spectrum of the proposed absorber as a function of polarization angle and frequency.

We have also investigated the omnidirectional characteristic for the proposed absorber. Figure 6 plots the absorption spectra of the absorber as function of frequency and incident angle for TE and TM polarization, respectively. The two absorption peaks can be observed within a wide range of incident angles for both TE and TM polarizations. Moreover, at the incident angle below $60^{\circ}$, a substantial overlap can be observed between the absorption spectra for both polarizations. Therefore, the proposed device can also be verified to be a non-polarization-dependent absorber within this range. 
(a)

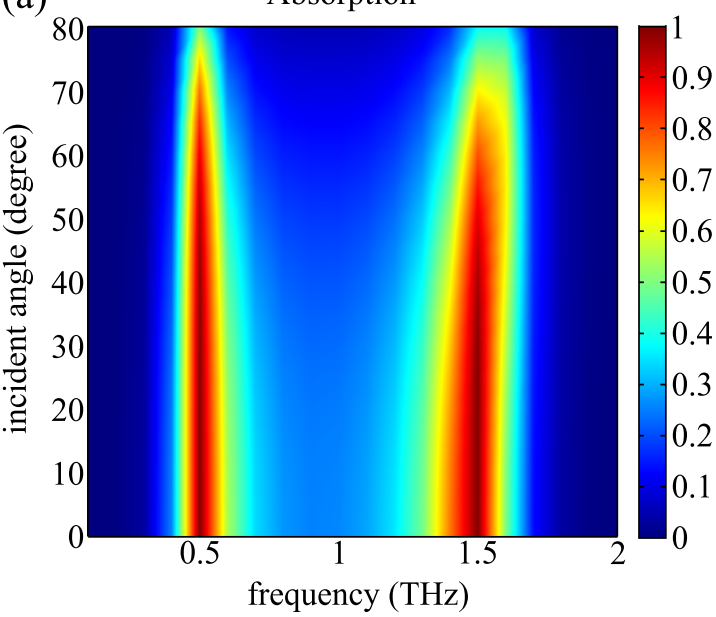

(b)

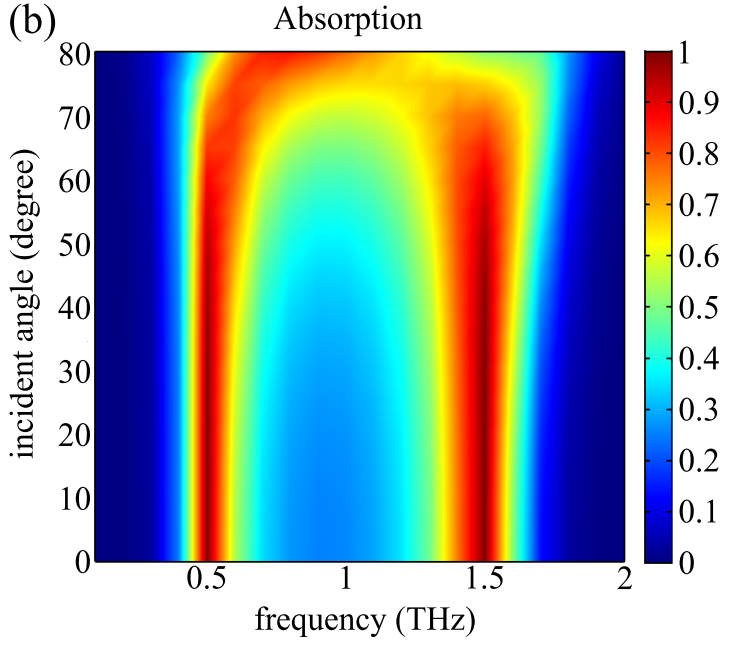

Figure 6. The absorption spectrum of the proposed absorber as a function of incident angle and frequency for (a) TM and (b) TE polarizations.

\section{Conclusions}

In summary, a dual-band perfect absorber based on graphene patches has been designed in the low terahertz regime by using impedance matching concept. First, we assigned an equivalent circuit to the absorber. We then adjusted the real part of the input admittance of the absorber to be close to the free space admittance and the imaginary part of the input admittance to be zero at two frequencies. In addition, the dielectric slab is considered as a quarter wavelength transmission line at the center of the two frequencies. This method resulted in closed-form relations for the geometry of the structure and the characteristics of the applied material. According to the results of simulation and analytical circuit model, the recommended absorber can operate with perfect absorption at $0.5 \mathrm{THz}$ and $0.15 \mathrm{THz}$. Further, the device are insensitive to polarization and omnidirectional.

Author Contributions: Formal analysis and writing-original draft preparation, Z.L.; investigation, L.G.; writing-review and editing, Q.Z. All authors have read and agreed to the published version of the manuscript.

Funding: This research was supported by the Key-Area Research and Development Program of Guangdong Province (2020B090922006), the Guangdong Science and Technology Department (2018B030323017), the Innovation Project of Graduate School of South China Normal University (2019LKXM010), and the National Key Research and Development Program of China (2017YFB1104500).

Acknowledgments: This research was supported by the Key-Area Research and Development Program of Guangdong Province (2020B090922006); the Guangdong Science and Technology Department (2018B030323017); the Innovation Project of Graduate School of South China Normal University (2019LKXM010); the National Key Research and Development Program of China (2017YFB1104500); and Guangdong Provincial Key Laboratory of Industrial Ultrashort Pulse Laser Technology, Shenzhen 518055, China.

Conflicts of Interest: The authors declare no conflict of interest.

\section{References}

1. Pendry, J.B.; Schurig, D.; Smith, D.R. Controlling Electromagnetic Fields. Science 2006, 312, $1780-1782$. [CrossRef] [PubMed]

2. Paggi, M. Singular, hypersingular and singular free electromagnetic fields at wedge tips in metamaterials. Int. J. Solids Struct. 2010, 47, 2062-2069. [CrossRef]

3. Tao, H.; Landy, N.I.; Bingham, C.M.; Zhang, X.; Averitt, R.D.; Padilla, W.J. A metamaterial absorber for the terahertz regime: Design, fabrication and characterization. Opt. Express 2008, 16, 7181-7188. [CrossRef]

4. Carranza, I.E.; Grant, J.; Gough J.; Cumming D.R.S. Metamaterial-Based Terahertz Imaging. IEEE Trans. THz Sci. Technol. 2015, 5, 892-901. [CrossRef] 
5. Cong, L.; Tan, S.; Yahiaoui, R.; Yan, F.; Zhang, W.; Singh, R. Experimental demonstration of ultrasensitive sensing with terahertz metamaterial absorbers: A comparison with the metasurfaces. Appl. Phys. Lett. 2015, 106, 031107. [CrossRef]

6. Sabah, C.; Mulla, B.; Altan, H.; Ozyuzer, L. Cross-like terahertz metamaterial absorber for sensing applications. Pramana 2018, 91, 17. [CrossRef]

7. He, Y.; Zhang, B.; He, T.; Chen, T.; Wang, G.; Hou, Y.; Xiong, W.; Shen, J. Optically-controlled metamaterial absorber based on hybrid structure. Opt. Commun. 2015, 356, 595-598. [CrossRef]

8. Huang, M.L.; Cheng, Y.Z.; Cheng, Z.Z.; Chen, H.R.; Mao, X.S.; Gong, R.Z. Based on graphene tunable dual-band terahertz metamaterial absorber with wide-angle. Opt. Commun. 2018, 415, 194-201. [CrossRef]

9. Zhang, J.G.; Tian, J.P.; Li, L. A Dual-Band Tunable Metamaterial Near-Unity Absorber Composed of Periodic Cross and Disk Graphene Arrays. IEEE Photonics J. 2018, 10, 4800512. [CrossRef]

10. Zhang, Y.B.; Cen, C.L.; Liang, C.P.; Yi, Z.; Chen, X.F.; Li, M.W.; Zhou, Z.G.; Tang, Y.J.; Yi, Y.G.; Zhang, G.F. Dual-band switchable terahertz metamaterial absorber based on metal nanostructure. Res. Phys. 2019, 14, 102422. [CrossRef]

11. Li, H.J.; Ji, C.S.; Ren, Y.Z.; Hu, J.G.; Qin, M.; Wang, L.L. Investigation of multiband plasmonic metamaterial perfect absorbers based on graphene ribbons by the phase-coupled method. Carbon 2019, 141, 481-487. [CrossRef]

12. Qi, L.M.; Liu, C.; Shah, S.M.A. A broad dual-band switchable graphene-based terahertz metamaterial absorber. Carbon 2019, 153, 179-188. [CrossRef]

13. Qi, Y.P.; Zhang, Y.; Liu, C.Q.; Zhang, T.; Zhang, B.H.; Wang, L.Y.; Deng, X.Y.; Wang, X.X.; Yu, Y. A Tunable Terahertz Metamaterial Absorber Composed of Hourglass-Shaped Graphene Arrays. Nanomaterials 2020, 10, 553. [CrossRef] [PubMed]

14. Cen, C.L.; Zhang, Y.B.; Chen, X.F.; Yang, H.; Yi, Z.; Yao, W.T.; Tang, Y.J.; Yi, Y.G.; Wang, J.Q.; Wu, P.H. A dual-band metamaterial absorber for graphene surface plasmon resonance at terahertz frequency. Phys. $E$ 2020, 117, 113840. [CrossRef]

15. Khavasi, A. Design of ultra-broadband graphene absorber using circuit theory. J. Opt. Soc. Am. B 2015, 32, 1941-1946. [CrossRef]

16. Yao, G.; Ling, F.R.; Yue, J.; Luo, C.Y.; Ji, J.; Yao, J.Q. Dual-band tunable perfect metamaterial absorber in the $\mathrm{THz}$ range. Opt. Express 2016, 24, 1518-1527. [CrossRef]

17. Barzegar-Parizi, S.; Khavasi, A. Designing Dual-Band Absorbers by Graphene/Metallic Metasurfaces. IEEE J. Quantum Electron. 2019, 55, 7300108. [CrossRef]

18. Barzegar-Parizi, S. Graphene-based tunable dual-band absorbers by ribbon/disk array. Opt. Quant. Electron. 2019, 51, 167. [CrossRef]

19. Singh, P.K.; Korolev, K.A.; Afsar, M.N.; Sonkusale, S. Single and dual band 77/95/110 ghz metamaterial absorbers on flexible polyimide substrate. Appl. Phys. Lett. 2011, 99, 264101. [CrossRef]

20. Jo, G.; Choe, M.; Lee, S.; Park, W.; Kahng, Y.H.; Lee, T. The application of graphene as electrodes in electrical and optical devices. Nanotechnology 2012, 23, 112001. [CrossRef]

21. Zhong, Y.J.; Zhen Z.; Zhu H.W. Graphene: Fundamental research and potential applications. FlatChem 2017, 4, 20-32. [CrossRef]

22. Ren, S.; Rong, P.; Yu, Q. Preparations, properties and applications of graphene in functional devices: A concise review. Ceram. Int. 2018, 44, 11940-11955. [CrossRef]

23. Coros, M.; Pogacean, F.; Magerusan, L.; Socaci, C.; Pruneanu, S. A brief overview on synthesis and applications of graphene and graphene-based nanomaterials. Front. Mater. Sci. 2019, 13, 23-32. [CrossRef]

24. Bonaccorso, F.; Sun, Z.; Hasan, T.; Ferrari, A. C. Graphene photonics and optoelectronics. Nat. Photonics 2010, 4, 611-622. [CrossRef]

25. Castro Neto, A.H.; Guinea, F.; Peres, N.M.R.; Novoselov, K.S.; Geim, A.K. The electronic properties of graphene. Rev. Mod. Phys. 2009, 81, 109-162. [CrossRef]

26. Wang, F.; Zhang, Y.; Tian, C.; Girit, C.; Zettle, A.; Crommie, M.; Shen, Y.R. Gate-variable optical transitions in graphene. Science 2008, 320, 206-209. [CrossRef]

27. Novoselov, K.S.; Fal'ko, V.I.; Colombo, L.; Gellert, P.R.; Schwab, M.G.; Kim, K. A roadmap for graphene. Nature 2012, 490, 192-200. [CrossRef]

28. Koppens, F.H.L.; Chang, D.E.; de Abajo, F.J.G. Graphene plasmonics: A platform for strong light-matter interactions. Nano Lett. 2011, 11, 3370-3377. [CrossRef] 
29. Grigorenko, A.N.; Polini, M.; Novoselov, K.S. Graphene plasmonics. Nat. Photonics 2012, 487, 749-758. [CrossRef]

30. Andryieuski, A.; Lavrinenko, A.V. Graphene metamaterials based tunable terahertz absorber: Effective surface conductivity approach. Opt. Express 2013, 21, 9144-9155. [CrossRef]

31. Barzegar-Parizi, S.; Tavakol, M.R.; Khavasi, A. Deriving Surface Impedance for 2-D Arrays of Graphene Patches Using a Variational Method. IEEE J. Quantum Electron. 2017, 53, 7000106. [CrossRef]

32. Llatser, I.; Kremers, C.; Cabellos-Aparicio, A.; Jornet, J.M.; Alarcon, E.; Chigrin, D.N. Graphene-based nano-patch antenna for terahertz radiation. Photonics Nanostruct. Fundam. Appl. 2012, 10, 353-358. [CrossRef]

33. Hanson, G.W. Dyadic Green's functions and guided surface waves for a surface conductivity model of graphene. J. Appl. Phys. 2008, 103, 064302. [CrossRef]

34. Sensale-Rodriguez, B.; Yan, R.S.; Kelly, M.M.; Fang, T.; Tahy, K.; Hwang, W.S.; Jena, D.; Liu, L.; Xing, H.G. Broadband graphene terahertz modulators enabled by intraband transitions. Nat. Commun. 2012, 3, 780. [CrossRef] [PubMed]

35. Luukkonen, O.; Simovski, C.; Granet, G.; Goussetis, G.; Lioubtchenko, D.; Räisänen, A.V.; Tretyakov, S.A. Simple and accurate analytical model of planar grids and high-impedance surfaces comprising metal strips or patches. IEEE Trans. Antennas Propag. 2008, 56, 1624-1632. [CrossRef]

36. Jang, M.S.; Brar, V.W.; Sherrott, M.C.; Lopez, J.J.; Kim, L.; Kim, S.; Choi, M.; Atwater, H.A. Tunable large resonant absorption in a mid-infrared graphene Salisbury screen. Phys. Rev. B 2014, 90, 165409. [CrossRef]

37. Kim, S.; Jang, M.S.; Brar, V.W.; Mauser, K.W.; Atwater, H.A. Electronically tunable perfect absorption in graphene. Nano Lett. 2018, 18, 971-979. [CrossRef] [PubMed]

38. Ju, L.; Geng, B.S.; Horng, J.; Girit, C.; Martin, M.; Hao, Z.; Bechtel, H.A.; Liang, X.G.; Zettl, A.; Shen, Y.R.; Wang, F. Graphene plasmonics for tunable terahertz metamaterials. Nat. Nanotechnol. 2011, 6, 630-634. [CrossRef]

39. Dean, C.R.; Young, A.F.; Meric, I.; Lee, C.; Wang, L.; Sorgenfrei, S.; Watanabe, K.; Taniguchi, T.; Kim, P.; Shepard, K.L.; Hone, J. Boron nitride substrates for high-quality graphene electronics. Nat. Nanotechnol. 2010, 5, 722-726. [CrossRef]

40. Bolotin, K.I.; Sikes, K.J.; Jiang, Z.; Klima, M.; udenberg, G.; Hone, J.; Kim, P.; Stormer, H.L. Ultrahigh electron mobility in suspended graphene. Solid State Commun. 2008, 146, 9-10. [CrossRef]

41. Barzegar-Parizi, S. Realization of wide-angle and wideband absorber using metallic and graphene-based metasurface for mid-infrared and low THz frequency. Opt. Quant. Electron. 2018, 50, 378. [CrossRef]

Sample Availability: No samples.

(C) 2020 by the authors. Licensee MDPI, Basel, Switzerland. This article is an open access article distributed under the terms and conditions of the Creative Commons Attribution (CC BY) license (http:/ / creativecommons.org/licenses/by/4.0/). 